\title{
A Study on the Safety of Percutaneous Tracheostomy in Patients with Severe Acute Respiratory Syndrome Novel Corona Virus 2 (SARS-nCoV2) Infection: A Single-Center Observational Cohort Study in a CoVID Intensive Care Unit
}

\author{
Krishna Kumar Mylavarapu ${ }^{1}$ (I) $\cdot$ Aditya Joshi $^{1} \cdot$ Ranjith Nair $^{2} \cdot$ Rangraj Setlur $^{3} \cdot$ Rajan Kapoor $^{2}$
}

Accepted: 9 June 2021 / Published online: 19 June 2021

(C) The Author(s), under exclusive licence to Springer Nature Switzerland AG 2021

\begin{abstract}
Background We studied the safety of percutaneous dilatational tracheostomy (PCDT) in severe acute respiratory syndrome novel coronavirus 2 (SARS-nCoV2).

Patients and Methods From 01 March 20 to 30 November 2020, 1635 required hospital admission of which 145 (9\%) required intensive (ICU) care. The primary outcomes are mortality and secondary outcomes were duration of invasive mechanical ventilation (IMV), length of stay (LOS) in ICU and hospital, and days required for decannulation.

Results Out of the 145 (9\%), 107 (73.7\%) were males (mean 61.4 years, median body mass index (BMI) of $28.2 \mathrm{~kg} / \mathrm{m}^{2}$ ), and 38 (26.2\%) were females (mean 58.10 years, median BMI of $31.2 \mathrm{~kg} / \mathrm{m}^{2}$ ). In the cohort of $80(55.17 \%)$ requiring IMV, $19(23.7 \%)$ died within 72 hours and were not included in the study, 37 (group "NT") and 24 (group "T") had a median duration of ventilation of $9 \mathrm{~d}$ (IQR, 6-11) and $12 \mathrm{~d}$ (IQR, 11-17.25) respectively. Patients in group "T" underwent PCDT based on clinical criteria (fraction of inspired oxygen $\left(\mathrm{FiO}_{2}\right)$ of $\leq 50 \%$ with positive end-expiratory pressure (PEEP) of $\leq 10 \mathrm{cms}$ of $\mathrm{H}_{2} \mathrm{O}$ with stable hemodynamics), and 16 (66.7\%) had survived. The reverse transcription-polymerase chain reaction (RT-PCR) does not need to be negative, and none of the health care workers (HCW's) were infected. The Cox-hazard ratio [HR] is $0.19,95 \%$ confidence interval $[\mathrm{CI}](0.09,0.41)$ with a P-value of $<0.001,83(57.2 \%)$ were discharged with a mortality of $42.8 \%$.

Conclusions PCDT is safe and effective in patients anticipated in need of prolonged mechanical ventilation.
\end{abstract}

Keywords COVID-19 · ARDS $\cdot$ Percutaneous tracheostomy

\section{Introduction}

During the period from 01 March to 30 November 2020, the SARS-nCoV2 pandemic patients admitted to the intensive care unit were studied. The hospital is a super-specialty tertiary care center located in eastern India with 900 hospital beds

This article is part of the Topical Collection on Covid-19

Krishna Kumar Mylavarapu

krishnakm312@gmail.com

Aditya Joshi

majoraditya@gmail.com

Ranjith Nair

ranjith3246@gmail.com

Rangraj Setlur

rangraj@gmail.com and 28 intensive care unit beds. Additionally, a 100-bedded CoVID-designated ward with 07 ICU beds was also started to meet the demand. Patients requiring intubation and invasive mechanical ventilation (IMV) had high mortality when compared to those who were not [1]. During the initial phase of the pandemic, percutaneous tracheostomy, considered as an

Rajan Kapoor

majrkapoor@gmail.com

1 Department of Anaesthesiology \& Critical Care, Command Hospital (EC), Kolkata 700027, India

2 Department of Internal medicine, Command Hospital (EC), Kolkata 700027, India

3 Department of Anaesthesiology \& Critical Care, Armed Forces Medical College, Pune, Maharashtra 411040, India 
aerosol-generating procedure (AGP), could not be done in patients requiring prolonged ventilation due to fear of the spread of infection to health care workers (HCW's) [2]. However, in the latter part of the pandemic, there was greater confidence in personal protective measures, and with an increased surge in the number of cases, and acute shortage of ICU beds, patients anticipated to have prolonged ventilation with improving ventilatory settings $\left(\mathrm{FiO}_{2} \leq 50 \%\right.$ and $\mathrm{PEEP} \leq$ $10 \mathrm{cms}$ of $\mathrm{H}_{2} \mathrm{O}$ with stable hemodynamics) were considered for percutaneous tracheostomy ( $\sim 7$ days) to reduce the ICU LOS and morbidity. The RT-PCR did not need to be negative before performing tracheostomy, and none of the HCWs were infected during the procedure. We studied the safety of percutaneous tracheostomy, and whether it improved the outcomes in patients in whom prolonged ventilation was anticipated, besides their comorbidities, demographic, and biochemical/ biomarkers characteristics.

\section{Materials and Methods}

\section{Patient Selection}

After approval from the hospital ethics committee, health records of the patients admitted to the CoVID intensive care unit with severe SARS-nCoV2 during the period from 01 March to 30 November 20 were collected retrospectively. Patients admitted to the intensive care unit had their diagnosis confirmed by RT-PCR using nasopharyngeal and throat swab and/or by radiological imaging (chest X-ray or by high-resolution computerized tomography (HRCT) of chest). We specifically studied the patients who underwent bedside percutaneous tracheostomy. All the patients had their informed consent taken before performing tracheostomy either from the patients themselves or by utilizing electronic/video conferencing from the patient's surrogate decision-makers. Expert consensus recommends that CoVID-19 patients, anticipated to have prolonged mechanical ventilation with minimal ventilatory settings, i.e., $\mathrm{FiO}_{2} \leq 50 \%$ and PEEP $\leq 10$, should undergo tracheostomy to reduce the duration of mechanical ventilation and ICU LOS [3-5]. Our institutional guidelines have similar inclusion criteria, in patients for whom meaningful recovery is expected. All procedures were done with strict adherence to personal protective measures against aerosol-generating procedures (AGPs) as defined by national / health ministry guidelines (personal protective equipment (PPE), FFP3 masks with fluid repellent gowns, gloves, and eye shield/glasses) and by the hospital infection control committee.

\section{Procedure}

After taking informed consent, anticoagulation was stopped 24 hours before the planned tracheostomy. We used the
Ciaglia Blue Rhino $® /$ Portex $®$ advanced percutaneous tracheostomy kit. Maximum sterile barrier precautions were used during the procedure. The size of the tracheostomy tube selected was $7.0 / 7.5 \mathrm{~mm}$ in females and $8.0 / 8.5 \mathrm{~mm}$ in adult male patients. Due to the limited availability of bronchoscopes and to reduce aerosol generation, modifications were made to the conventional percutaneous tracheostomy in the CoVID ICU. Complete neuromuscular paralysis with sedation and analgesia was used to stop the patient from coughing. Once the patient was adequately positioned with neck extension, ultrasound (USG) was used to identify the cervical vascular structures. The $\mathrm{FiO}_{2}$ was increased to $100 \%$ and the PEEP was set at $5 \mathrm{cms}$ of $\mathrm{H}_{2} \mathrm{O}$. After identifying the anatomical landmarks, local infiltration was done using $2 \%$ lignocaine with adrenaline $(1: 200,000)$. A 2-to-3 cm horizontal incision was made over the anterior part of the neck overlying the trachea below the cricoid cartilage or 2 finger breadths above the manubrium sternum. Blunt dissection of the pre-tracheal tissue was done until the trachea was palpable. Once the trachea was identified, a modified Seldinger's technique was used, followed by "single stage" dilation using the "rhino" for placing the appropriate size tracheostomy tube $[5,6]$. The presence of end-tidal carbon dioxide confirmed the correct tube placement, along with clinical auscultation of the chest.

To minimize aerosol generation, a bronchoscope was used only, if there, was any doubt in tube position. No hemodynamic/hypoxic episodes were seen in our study. In the post-procedure period to minimize the aerosol generation, the cuff was kept inflated and a closed-suction catheter was used for clearing the airway. The deflation of the cuff, replacement of the tracheostomy tube, and initiating a plan for decannulation was deferred until the results of the RT-PCR turned negative. There were no major complications of note in any of our cases.

\section{Statistical Analysis}

The continuous variables are presented as median (interquartile range $[\mathrm{IQR}]$ ), whereas categorical variables were expressed as percentages (\%). Statistical analyses are done using " $R$ " 3.6.2 software. For significance estimation, the student t-test was used for continuous variables and the Chisquare test for categorical data. Kaplan-Meier estimator and Cox's proportional hazard ratio were used for survival analysis.

\section{Results}

\section{Characteristics and Treatment}

From 01 March to 30 November 20, 1635, CoVID-19 patients required hospital admission as per the WHO ordinal scale [7]. 
Table 1 Baseline demographic characteristics (Signs \& symptoms, Comorbidities and laboratory findings)

\begin{tabular}{|c|c|c|c|c|}
\hline $\begin{array}{l}\text { Clinical } \\
\text { Characteristics }\end{array}$ & $\begin{array}{l}\text { All patients } \\
\mathrm{n}=145\end{array}$ & $\begin{array}{l}\text { Survivors } \\
\mathrm{n}=83\end{array}$ & $\begin{array}{l}\text { Non survivors } \\
n=62\end{array}$ & $\mathrm{P}$ value \\
\hline $\begin{array}{l}\text { Age, mean(range) } \\
\text { Age, median (IQR) }\end{array}$ & $\begin{array}{l}60.4(20-95) \\
62(52-71)\end{array}$ & $\begin{array}{l}58.6(20-88) \\
62(49.5-69)\end{array}$ & $\begin{array}{l}62.6(26-95) \\
64.5(52.5-73.7)\end{array}$ & 0.10 \\
\hline Sex, M & $107(73.8)$ & $66(61.7)$ & $41(38.3)$ & 0.06 \\
\hline \multicolumn{5}{|l|}{ Signs \& symptoms } \\
\hline Fever & $102(70.3)$ & $54(52.9)$ & $48(47.1)$ & 0.10 \\
\hline Cough & $61(42)$ & $39(63.9)$ & $22(36.1)$ & 0.165 \\
\hline Dyspnea & $126(86.9)$ & $70(55.5)$ & $56(44.4)$ & 0.29 \\
\hline Diarrhoea & $31(21.3)$ & $15(48.3)$ & $16(51.6)$ & 0.26 \\
\hline \multicolumn{5}{|l|}{ Comorbidities } \\
\hline Diabetes & $41(28.2)$ & $18(43.9)$ & $23(56.1)$ & 0.04 \\
\hline Hypertension & $49(33.7)$ & $24(48.9)$ & $25(51.1)$ & 0.15 \\
\hline CKD & $19(13.1)$ & $07(36.8)$ & $12(63.1)$ & 0.08 \\
\hline CVA & $14(9.6)$ & $6(42.8)$ & $8(57.1)$ & 0.27 \\
\hline CAD & $17(11.7)$ & $7(41.10$ & $10(58.8)$ & 0.19 \\
\hline \multicolumn{5}{|l|}{ Lab findings } \\
\hline $\mathrm{Hb}, \mathrm{gm} / \mathrm{dL}$ & $11(9.7-12.4)$ & $11.2(9.5-12.4)$ & $10.7(9.9-12.5)$ & 0.88 \\
\hline $\mathrm{TLC} \times 10^{9} / \mathrm{L}$ & $9.6(6.7-15.2)$ & $8.6(6.2-14)$ & $10.6(7.7-16.3)$ & 0.03 \\
\hline Plt X $10^{9} / \mathrm{L}$ & $150(106-224)$ & $148(102-256)$ & $150(110-204)$ & 0.06 \\
\hline NLR & $9.4(4.6-17.6)$ & $8.9(5-17)$ & $9.9(4-17.6)$ & 0.62 \\
\hline LDH (IU/L) & $864(574-1512)$ & $812(576-1048)$ & $987(570-1867)$ & 0.009 \\
\hline Ferritin (upper limit till $1200 \mathrm{ng} / \mathrm{ml}$ ) & $639(263-897)$ & $692(357-934)$ & $639(135-883)$ & 0.19 \\
\hline D-Dimer & $2944(750-7407)$ & 1091(750-12000) & $4547(1950-19000)$ & $<0.001$ \\
\hline
\end{tabular}

Out of these, 145 patients with severe SARS-nCoV2 pneumonia required intensive care with an ordinal scale of 5-7 (Table 1). Males and females constituted 107 (73.8\%) and $38(26.3 \%)$ of the total, with a mean age of 61.4 and 58.10 years respectively. There was no significant association between age and the outcomes. However, on subgroup analysis, in the non-survivor group, males were significantly older, when compared to females. The median BMI in males and females was $28.2 \mathrm{~kg} / \mathrm{m}^{2}$ and $31.2 \mathrm{~kg} / \mathrm{m}^{2}$ respectively with younger obese females showing increased mortality. However, this analysis could be limited due to the underrepresentation $(26.3 \%)$ of females in the group. Eighty-three $(57.2 \%)$ patients survived and were discharged to home while $62(42.8 \%)$ patients died.

The most common presenting symptoms were fever, cough, dyspnea, and diarrhea. These symptoms were distributed equally among survivors and non-survivors. Primary hypertension was the commonest comorbidity which was seen in $33.7 \%$ of cases, followed by Type- 2 diabetes mellitus in 28.2 $\%$ of cases. The presence of Type- 2 diabetes mellitus was significantly higher in non-survivors $(37.1 \%)$ compared to survivors $(21.6 \%)(\mathrm{P}=0.04)$. The presence of two or more comorbidities was significantly more common in the nonsurvivor group (P 0.039) (Fig. 1). Among the laboratory investigations, neutrophilia with lymphocytopenia (high NLR ratio), high serum lactate dehydrogenase (LDH), and DDimer levels (survivors IQR, 750-12000 vs non-survivors IQR,1950-19000) had a significant impact on the mortality

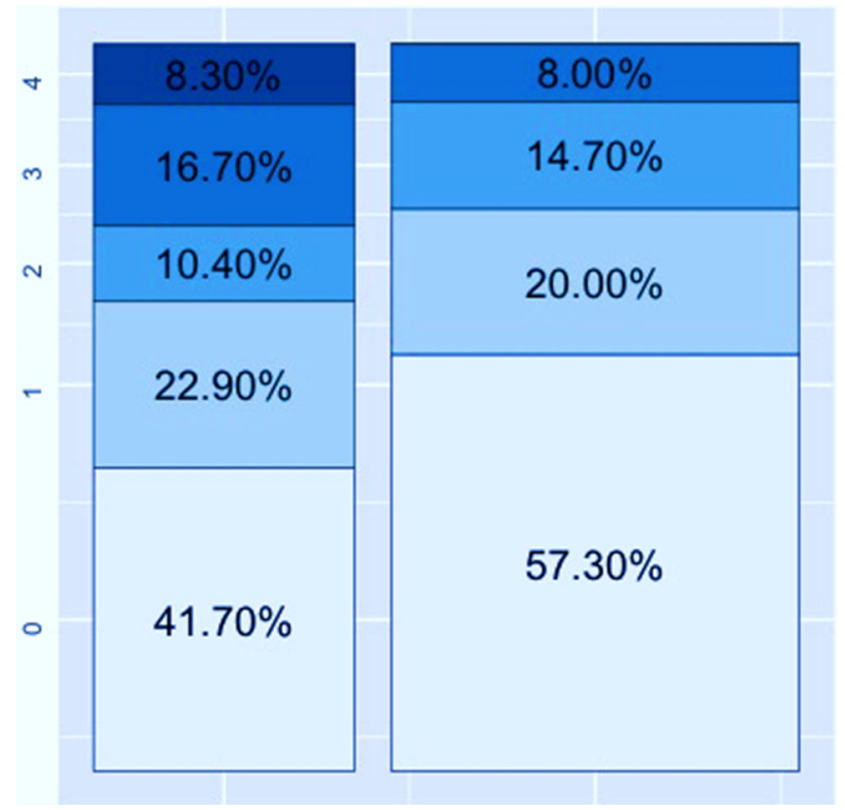

Figure 1 Effect of number of comorbidities on overall outcome. Nonsurvivor group (left side of bargraph) had significantly higher number of patients with multiple comorbidities $(\mathrm{p}=0.039)$ 
among the non-survivors. A total of 17 patients (11.7\%) required renal replacement therapy. Seventy-nine $(54.4 \%)$ patients required vasopressor support.

\section{Respiratory Support}

Depending on the severity of hypoxia, patients were managed with non-rebreathing mask (NRBM), high flow nasal oxygen (HFNO), or non-invasive ventilation (NIV). We encouraged and motivated voluntary self-awake proning in non-intubated patients who were cooperative. Twenty-eight patients (19.3\%) required supplemental oxygen by non-rebreathing mask (NRBM) at $15 \mathrm{l} / \mathrm{min}$ of oxygen; of these, 21 patients (75\%) deteriorated and subsequently required intubation and mechanical ventilation. Fifty-four (37.2\%) patients were initially managed on high flow nasal oxygen therapy (HFNO); of these, 24 patients (44.4\%) also required invasive mechanical ventilation, and $63(43.4 \%)$ patients required non-invasive ventilation (NIV) respiratory support; of these, 35 (55.6\%) progressively worsened requiring invasive mechanical ventilation support. The timing of intubation in patients who were on NRBM/HFNO or NIV was left to the discretion of the bedside treating clinician. $80(55.1 \%)$ of 145 patients required invasive mechanical ventilation (IMV).

We used lung-protective ventilation (LPV) strategies with sedation and neuromuscular paralysis. Patients were prone to ventilate for 18-24 hours every day with at least 4-5 proning sessions unless contraindicated.

The mean time from the onset of illness to intubation and hospital admission to intubation was 10 and 9 days respectively. The mean $\mathrm{PaO} 2 / \mathrm{FiO} 2(\mathrm{P} / \mathrm{F})$ ratio and positive-end expiratory pressure (PEEP) at the time of intubation was 140 and 10 cms of $\mathrm{H} 2 \mathrm{O}$ respectively in both groups. Three patients (8.1 $\%$ ) in the group "T" and one (2.7\%) patient in the group "NT" were on veno-venous extracorporeal membrane oxygenation (VV-ECMO). Nineteen (23.7\%) of 80 patients on invasive mechanical ventilation had delayed presentation with sepsis and multi-organ failure and died within 48-72 hours of ICU admission. Thirty-seven (46.2\%) in the group "NT" patients were on a mechanical ventilator with a median duration of $9 \mathrm{~d}$ (IQR, 6-11) and 24 (30\%) patients in the group " $\mathrm{T}$ " with a median duration of $12 \mathrm{~d}$ (IQR, 11-17.25). Primary extubation was considered whenever feasible with $2(7.6 \%)$ patients in group "NT" meeting the criteria for extubation. Twenty-four $(30 \%)$ patients in group " $\mathrm{T}$ " did not meet the extubation criteria and underwent percutaneous tracheostomy. The median time for tracheostomy was 7 (IQR, 3-11) days. Eighteen (75\%) of 24 patients in the group " $\mathrm{T}$ " who underwent tracheostomy survived and were discharged from the hospital. The ICU length of stay was 7 days (IQR, 1-24), and the hospital length of stay was 14 days (IQR, 2-25). The median time for tracheostomy decannulation was 16 days (IQR, 8-34). None of the health care workers (HCWs) were infected during the procedure. We had 3 minor complications post-tracheostomy in the group " $T$ ": (a) bleeding seen in one patient with chronic kidney disease and (b) subcutaneous emphysema in two patients with pre-existing pneumomediastinum on chest CT scan. All were managed conservatively. None of them required any further intervention.

Three patients on VV-ECMO in the group "T" underwent percutaneous tracheostomy without any complications. Decannulation was not a prerequisite criterion for transferring patients out of the CoVID ICU. All patients who turned SARS-nCoV2 negative were shifted to Non-CoVID intensive care unit/acute wards for monitoring and decannulation was done as per the clinical criteria [8].

\section{Clinical Course and Outcomes}

Patients who underwent early tracheostomy had a longer median duration of stay in the ICU compared to the nontracheostomy group (Fig. 2). The time-to-event calculated in patients who did not undergo tracheostomy and those who underwent tracheostomy by the Cox-hazard ratio [HR] is 0.19 with $95 \%$ confidence interval $[\mathrm{CI}](0.09,0.41)$ with a P-value $<0.001$ (Fig. 3), suggesting that early tracheostomy may improve the survival outcomes in carefully selected patients with CoVID pneumonia. The log-rank test using the Kaplan-Meier estimator in patients who underwent tracheostomy had a better survival (Fig. 3). Out of 24 patients who underwent tracheostomy in CoVID ICU, 16 (66.6\%) patients were successfully decannulated subsequently and had a favorable outcome.

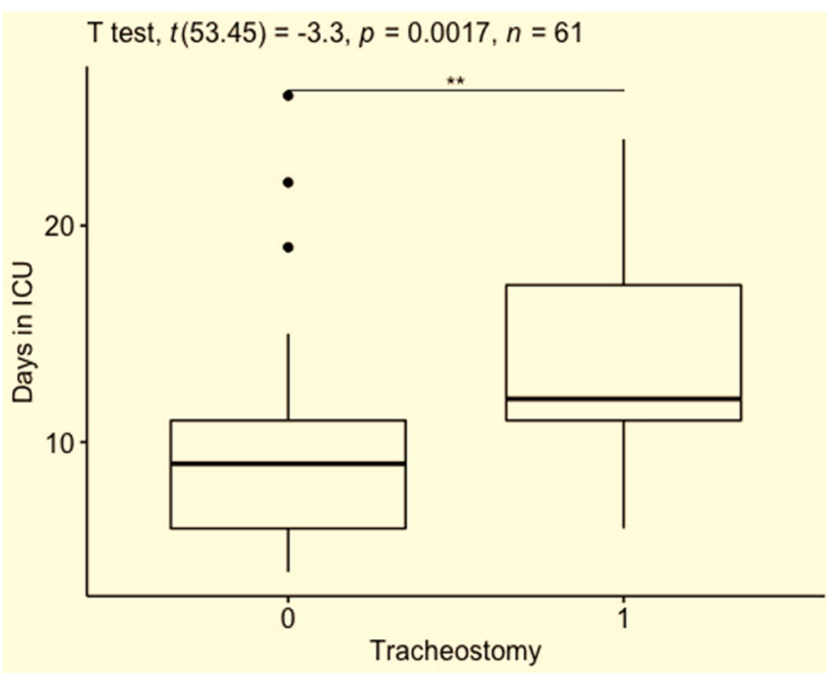

Figure 2 Outcomes of tracheostomy in covid ICU $(0 \rightarrow$ no tracheostomy, $1 \rightarrow$ tracheostomy) 


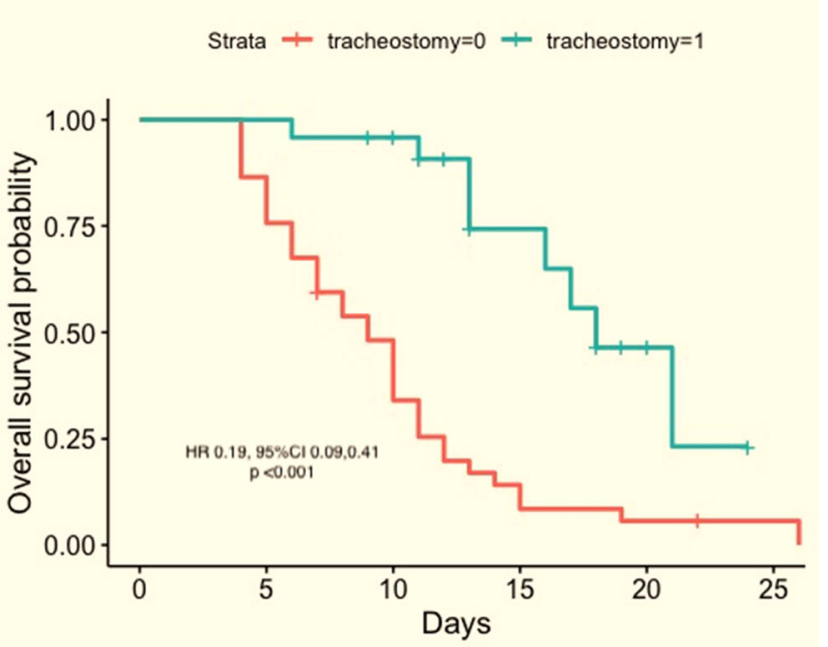

Figure 3 Kaplan Meir (KM) curve of patients on ventilator, staying in ICU for more than 03 days $(n=61)$, stratified according to tracheostomy. Green line depicts patients who

\section{Discussion}

In this single-center retrospective cohort study, 83/145 (57.2\%) patients with severe CoVID pneumonia were successfully discharged from ICU. Age has been reported as the single most important determinant of outcome in severe SARS-nCoV2 pneumonia; however, in our study, age was not associated with increased mortality [9]. On subgroup analysis though, to our surprise, we discovered that younger females with obesity had increased mortality when compared to males. This analysis could be limited due to the underrepresentation $(26.3 \%)$ of females in the group. However, worldwide, the incidence of mortality was higher in elderly males with multiple comorbidities compared to females infected with the SARS-nCoV2 virus $[10,11]$. There was no significant correlation between the duration of the symptoms and clinical outcomes in this study [12]. The impact of comorbidities on mortality was similar to other available data with higher mortality seen in patients with multiple comorbidities [1]. The laboratory parameters that were shown to predict increased mortality in severe SARS-nCoV2 pneumonia were high total leucocyte count (TLC), increased neutrophil-lymphocyte (NLR) ratio, high LDH, D-dimer, and ferritin levels which matches the findings of most studies from around the world [13]. Depending on the degree of severity of hypoxia, patients were managed with various oxygen delivery devices (e.g., NRBM, HFNO, and NIV). Awake, non-intubated, cooperative patients were encouraged and motivated for voluntary selfawake proning which was a pragmatic and effective method in improving oxygenation levels [14]. The exact timing of intubation and invasive mechanical ventilation on mortality in CoVID19 patients is not clear [15]. The mean $\mathrm{P} / \mathrm{F}$ ratio and PEEP in this study were similar to other studies [16]. The overall mortality in ventilated patients in our study was $42.8 \%$ which is similar to that seen in current literature (ranging from 40 to $88 \%$ ) $[17,18]$ and was attributable to nosocomial infections like ventilatorassociated pneumonia (VAP), and other secondary bacterial infections, resulting in multi-organ failure and death [16]. We feel that patients requiring prolonged mechanical ventilation with stable hemodynamics and improving ventilatory settings with $\mathrm{FiO} 2 \leq 50 \%$ and $\mathrm{PEEP} \leq 10$ should be considered for a bedside percutaneous tracheostomy to reduce the incidence of nosocomial infections and duration of mechanical ventilation.

Tracheostomy being an aerosol-generating procedure, there was always a fear of infections to the HCWs. However, the RT-PCR did not need to be negative before performing a tracheostomy [5]. During the latter part of the pandemic, there was greater confidence in personal protective measures being utilized and with the surge in the number of patients requiring ICU care and limited bed availability, percutaneous tracheostomy $(\sim 7$ days) was considered in a select group of patients based on the clinical criteria, i.e., $\mathrm{FiO}_{2} \leq 50 \%$ and $\mathrm{PEEP} \leq 10 \mathrm{cms}$ of $\mathrm{H}_{2} \mathrm{O}$ and stable hemodynamics irrespective of their APACHE II scores [3-5]. This allowed us to decrease the sedation levels, perform early ambulation, better tracheal toileting, and chest physiotherapy besides continue with oral feeds, thus reducing the ICU LOS, duration of the mechanical ventilation, and morbidity as was seen in other studies [3]. Our study showed that the percutaneous tracheostomy can be safely performed in patients with severe CoVID pneumonia requiring prolonged mechanical ventilation without any additional risk to the health care workers. It may be a reasonable approach to perform open surgical tracheostomy in Covid-19 pneumonia patients to mitigate aerosol generation; however, due to logistic reasons and to minimize the health care resource utilization, we choose percutaneous methods [19]. No healthcare worker transmissions resulted from performing the procedure and it must be considered in those with improving ventilatory settings.

\section{Limitations}

This is a retrospective comparative trial with limited sample size. The historic cohort group "NT" was populated by 35 (43.7\%) patients who could not be tracheostomized in the early phase of the pandemic due to the fear of infecting the health care workers. The cohort "T" group of 24 (30\%) patients underwent tracheostomy in the later part of the pandemic, by when the protocols had evolved, and there was greater confidence in the critical care team regarding personal protection and the procedure to be followed. There is no head-to-head comparison of the groups. Though our study showed improved outcomes in 16 (66.7\%) patients who underwent a percutaneous tracheostomy, the caveat was they were improving on the ventilatory parameters. Prospective randomized controlled studies are needed to fully ascertain the role of early tracheostomy (7 days) in the selected group of CoVID-19 patients with anticipated prolonged mechanical ventilation in limited-resource settings. 


\section{Conclusions}

Patients with severe SARS-nCoV2 pneumonia requiring intubation and invasive mechanical ventilation may progress to lung fibrosis requiring long-term mechanical ventilation and ICU care [20]. Our study has shown that a decision to perform percutaneous bedside tracheostomy ( $\sim 7$ days) over open methods seemed to be feasible, safe, and effective in the CoVID ICU, and could be considered in the treatment algorithm in patients anticipated to need prolonged mechanical ventilation to reduce the burden on the critical care facilities in resource-limited settings [5].

Code Availability Not applicable

Authors' Contributions Directly involved in collecting, editing, and analysis of the data.

Data Availability Available with the corresponding author

\section{Declaration}

Conflicts of interest/Competing interests The authors declare no competing interests.

Ethics approval (include appropriate approvals or waivers) Institutional Ethical committee approval taken

Consent to participate Informed Consent was taken from all the participants

Consent for publication Taken from the Head of the institution

\section{References}

1. Richardson S, Hirsch JS, Narasimhan M, Crawford JM, McGinn T, Davidson KW, et al. Presenting characteristics, comorbidities, and outcomes among 5700 patients hospitalized with COVID-19 in the New York City area. JAMA - J Am Med Assoc. 2020;323(20):2052-9.

2. Miles BA, Schiff B, Ganly I, Ow T, Cohen E, Genden E, et al. Tracheostomy during SARS-CoV-2 pandemic: recommendations from the New York Head and Neck Society. Head Neck. 2020;42(6):1282-90.

3. Breik O, Nankivell P, Sharma N, Bangash MN, Dawson C, Idle M, et al. Safety and 30-day outcomes of tracheostomy for COVID-19: a prospective observational cohort study. Br J Anaesth [Internet]. 2020;125(6):872-9. Available from. https://doi.org/10.1016/j.bja. 2020.08 .023 .

4. Lamb CR, Desai NR, Angel L, Chaddha U, Sachdeva A, Sethi S, et al. Use of tracheostomy during the COVID-19 pandemic: American College of Chest Physicians/American Association for Bronchology and Interventional Pulmonology/Association of Interventional Pulmonology Program Directors Expert Panel Report. Chest [Internet]. 2020;158(4):1499-514. Available from. https://doi.org/10.1016/j.chest.2020.05.571.
5. Takhar A, Walker A, Tricklebank S, Wyncoll D, Hart N, Jacob T, et al. Recommendation of a practical guideline for safe tracheostomy during the COVID-19 pandemic. Eur Arch Oto-RhinoLaryngology [Internet]. 2020;277(8):2173-84. Available from. https://doi.org/10.1007/s00405-020-05993-x.

6. Mcgrath BA, Brenner MJ, Warrillow SJ, Pandian V, Arora A, Cameron TS, et al. Health-care development tracheostomy in the COVID-19 era: global and multidisciplinary guidance. 1918;717-25.

7. World Health Organization. WHO R\&D Blueprint novel Coronavirus COVID-19 Therapeutic Trial Synopsis. World Heal Organ [Internet]. 2020;(February 18, 2020, Geneva, Switzerland): 1-9. Available from: http://www.moh.gov.sa/en/CoronaNew/ PressReleases/Pages/default.aspx

8. Singh RK, Saran S, Baronia AK. The practice of tracheostomy decannulation-a systematic review. J Intensive Care. 2017;5(1).

9. Bonanomi E, Cabrini L, Carlesso E, Castelli G, Cattaneo S, Cereda $\mathrm{D}$, et al. Risk factors associated with mortality among patients with COVID-19 in intensive care units in Lombardy. Italy. 2020;180(10): 1345-55.

10. Mukherjee V, Toth AT, Fenianos M, Martell S, Karpel HC, Postelnicu R, et al. Clinical outcomes in critically ill coronavirus disease 2019 patients: a unique New York City public hospital experience. Crit Care Explor. 2020;2(8):e0188.

11. Zangrillo A, Beretta L, Scandroglio AM, Monti G, Fominskiy E, Colombo S, et al. Characteristics, treatment, outcomes and cause of death of invasively ventilated patients with COVID-19 ARDS in Milan. Italy. 2020:1-12.

12. Larsson E, Brattström O, Agvald-Öhman C, Grip J, Campoccia Jalde F, Strålin K, et al. Characteristics and outcomes of patients with COVID-19 admitted to ICU in a tertiary hospital in Stockholm, Sweden. Acta Anaesthesiol Scand. 2020;(September).

13. Frater JL, Zini G, Onofrio G, Rogers HJ. COVID-19 and the clinical hematology laboratory. 2020;42(April):11-8.

14. Coppo A, Bellani G, Winterton D, Di Pierro M, Soria A, Faverio P, et al. Feasibility and physiological effects of prone positioning in non-intubated patients with acute respiratory failure due to COVID19 (PRON-COVID): a prospective cohort study. Lancet Respir Med [Internet]. 2020;8(8):765-74. Available from. https://doi.org/ $10.1016 / \mathrm{S} 2213-2600(20) 30268-\mathrm{X}$.

15. Hernandez-romieu AC, Adelman MW, Hockstein MA, Robichaux CJ, Edwards JA, Fazio JC, et al. Timing of intubation and mortality among critically ill coronavirus disease 2019 patients: a singlecenter cohort study. 2020;1-9.

16. Id CSK, Sahjwani D, Brown AW, Feroz S, Cameron P, Id EO, et al. Outcomes of mechanically ventilated patients with COVID-19 associated respiratory failure. 2020;1-9. Available from: https://doi. org/10.1371/journal.pone.0242651

17. Armstrong RA, Kane AD, Cook TM. Outcomes from intensive care in patients with COVID-19: a systematic review and metaanalysis of observational studies. Anesthesia. 2020;75(10):1340-9.

18. Quah P, Li A, Phua J, Phua J. Mortality rates of patients with COVID-19 in the intensive care unit: a systematic review of the emerging literature. Crit Care. 2020;24(1):1-4.

19. Chao TN, Harbison SP, Braslow BM, Hutchinson CT, Rajasekaran $\mathrm{K}$, Go BC, et al. Outcomes after tracheostomy in COVID-19 patients. Ann Surg. 2020;272(3):e181-6.

20. Ojo AS, Balogun SA, Williams OT, Ojo OS. Review article pulmonary fibrosis in COVID-19 survivors: predictive factors and risk reduction strategies. 2020;2020.

Publisher's Note Springer Nature remains neutral with regard to jurisdictional claims in published maps and institutional affiliations. 